
\title{
Nano-imaging of strain-tuned stripe textures in a Mott crystal
}

\author{
A. S. McLeod $\mathbb{D}^{1,4 凶}$, A. Wieteska ${ }^{1,4}$, G. Chiriaco ${ }^{1}$, B. Foutty ${ }^{1}$, Y. Wang ${ }^{2}$, Y. Yuan ${ }^{2,3}$, F. Xue ${ }^{3}$, V. Gopalan $\mathbb{E}^{2,3}$, L. Q. Chen $\mathbb{E}^{3}$, Z. Q. Mao $\mathbb{E}^{2}$,
} A. J. Millis ${ }^{1}$, A. N. Pasupathy $\mathbb{( D )}^{1 凶}$ and D. N. Basov $\mathbb{D I}^{1}$

The $4 d$ transition metal perovskites $\mathrm{Ca}_{n+1} \mathrm{Ru}_{n} \mathrm{O}_{3 n+1}$ have attracted interest for their strongly interacting electronic phases showing pronounced sensitivity to controllable stimuli like strain, temperature, and even electrical current. Through multi-messenger lowtemperature nano-imaging, we reveal a spontaneous striped texture of coexisting insulating and metallic domains in single crystals of the bilayer ruthenate $\mathrm{Ca}_{3}\left(\mathrm{Ti}_{x} \mathrm{Ru}_{1-x}\right)_{2} \mathrm{O}_{7}$ across its first-order Mott transition at $T \approx 95 \mathrm{~K}$. We image on-demand anisotropic nucleation and growth of these domains under in situ applied uniaxial strain rationalized through control of a spontaneous JahnTeller distortion. Our scanning nano-susceptibility imaging resolves the detailed susceptibility of coexisting phases to strain and temperature at the transition threshold. Comparing these nano-imaging results to bulk-sensitive elastoresistance measurements, we uncover an emergent "domain susceptibility" sensitive to both the volumetric phase fractions and elasticity of the self-organized domain lattice. Our combined susceptibility probes afford nano-scale insights into strain-mediated control over the insulator-metal transition in $4 \mathrm{~d}$ transition metal oxides.

npj Quantum Materials (2021)6:46; https://doi.org/10.1038/s41535-021-00339-0

\section{INTRODUCTION}

Many of the most exotic physical phenomena manifesting in the solid state are expressed in transition metal oxides, where the interaction of electronic, spin, and lattice degrees of freedom produce "soft" electronic states easily modified by external perturbations ${ }^{1,2}$. Celebrated hosts for these highly tunable phenomena include copper oxide high- $T_{c}$ superconductors, colossal magnetoresistive manganites ${ }^{3}$, polar materials like ferroelectrics and ferromagnets ${ }^{4}$, and Mott insulators poised near an insulator-metal transition (IMT) ${ }^{5}$. "Passive" strain from substrate epitaxy has emerged as an ubiquitous platform for tuning these phases in oxide thin films, affording stabilization of superconductivity $^{6,7}$ and ferroelectricity ${ }^{7,8}$ while abundantly tuning the insulator-metal transition among classes of correlated oxides like vanadates $^{9-11}$, nickelates ${ }^{12}$, ruthenates $^{13,14}$, and manganites ${ }^{15-17}$. However, the competing influences of reduced dimensionality and crystalline/stoichiometric defects motivate analogous studies of pristine single crystals to uncover fundamental mechanisms for strain-modulated control in these compounds; studies under uniaxial strain also afford access to anisotropic susceptibilities unresolvable under hydrostatic pressure. "Active" strain control through direct mechanical actuation of phase transitions in single crystals $s^{18-21}$ including the insulator-metal transition of $3 \mathrm{~d}$ metal oxides $^{9,22}$ can now provide a wealth of insights into the elastic susceptibility of functional electronic phases. This approach has recently been leveraged to explore lattice coupling of competing phases in the $4 d$ ruthenates ${ }^{23,24}$. Intrinsic predisposition of these systems towards coexistence of competing phases, especially in the case of functionally triggered first-order phase transitions, now motivates the application of microscopic probes capable of discerning strain susceptibilities arising from individual phases. In this work we distinguish such "intrinsic" susceptibility from "emergent" susceptibility associated with textured phase coexistence, a phenomenon owing generically to strain-coupling of phases observed e.g. in many IMTs ${ }^{17,25-28}$. Here we demonstrate the emergence of an elastic susceptibility associated directly with domain configurations of phases coexisting in a spontaneous nano-texture across the IMT of a prototypic Mott crystal- $\mathrm{Ti}$ substituted $\mathrm{Ca}_{3}\left(\mathrm{Ti}_{\mathrm{x}} \mathrm{Ru}_{1-\mathrm{x}}\right)_{2} \mathrm{O}_{7}$ (hereafter denoted $\mathrm{CTRO}$ ).

The parent bilayer perovskite ruthenate $\mathrm{Ca}_{3} \mathrm{Ru}_{2} \mathrm{O}_{7}$ (CRO) exhibits an IMT at $T_{\mathrm{IMT}}=48 \mathrm{~K}$, although with a directionally gapped Fermi surface surviving to low temperatures ${ }^{29,30}$ characterized by antiferromagnetically coupled ferromagnetic bilayers. This "soft" IMT is ascribed to a Peierls-like charge density wave instability presaged by several competing antiferromagnetic phases. On the other hand, substituting Ru by $3 d$ metals like $\mathrm{Ti}$ can produce an IMT completely distinct from that in CRO. For instance, Ti substitution at $x>4 \%$ induces a non-Fermi surfacedriven magnetic instability to G-type antiferromagnetic ground state, characterized by a gapped band structure along all axes ${ }^{31,32}$ whereby $T_{\text {IMT }}$ increases with substitution level ${ }^{32}$. This first-order IMT, identified by a hysteretic jump in resistivity over four orders of magnitude wide, could not be predicted at the level of singleparticle density functional theory ${ }^{31}$, implicating Mott physics. Moreover, that $\mathrm{Ti}^{4+}$ and $\mathrm{Ru}^{4+}$ cations have similar ionic radii rules out trivial origins for the IMT based on substitutional lattice distortion. Rather, the imposition of localized $\mathrm{Ti} t_{2 g}$ orbitals and associated removal of itinerant states near the Fermi level is expected to scatter itinerant carriers, as observed directly by STM probes of quasiparticle scattering in related Ti-substituted strontium ruthenates ${ }^{33,34}$. These scattering centers likely effect band-narrowing in the $\mathrm{Ru}$ orbital manifold ${ }^{31,32}$, suggesting a bandwidth-controlled Mott transition. The other factor favoring the Mott transition is the structural distortion induced by $\mathrm{Ti}$ doping; the Ti-doped samples exhibiting the Mott transition were found to show more flattened $\mathrm{RuO}_{6}$ octahedra in the paramagnetic state as compared to pristine $\mathrm{Ca}_{3} \mathrm{Ru}_{2} \mathrm{O}_{7}{ }^{35}$. This distortion predisposes CTRO towards a symmetry-preserving $\left(\mathrm{Bb} 2{ }_{1} \mathrm{~m}\right.$ space group) - and thus "coelastic" ${ }^{36}$ - orthorhombic structural transition from the high- $T$ metallic (" $L$ ") phase with comparatively long

\footnotetext{
${ }^{1}$ Dept. of Physics, Columbia University, 538 West 120th Street, New York, NY, USA. ${ }^{2}$ Dept. of Physics, Penn State University, University Park, PA, USA. ${ }^{3}$ Dept. of Materials Science \& Engineering, Penn State University, 221 Steidle Building, University Park, PA, USA. ${ }^{4}$ These authors contributed equally: A. S. McLeod, A. Wieteska. ${ }^{\circledR}$ email: am4734@columbia.edu; apn2108@columbia.edu
} 
$c$-axis to a shorter $c$-axis (" $\mathrm{S}$ ") insulating phase at low- $T$. The uncommonly large spontaneous strains of $+1.1 \%$ and $-0.85 \%$ along $b$ - and $c$-axes, respectively, in $x=3 \% \mathrm{CTRO}^{31}$ are almost an order of magnitude enhanced compared to the IMT of pure CRO ${ }^{37}$. At the level of electronic structure, this flattening distortion was implicated in lifting the degeneracy of $x y$ and $x z / y z$ orbitals in the $\mathrm{Ru} t_{2 g}$ manifold, inducing an orbital polarization essential for stabilizing the Mott insulating phase ${ }^{35,38}$. In fact, ab initio calculations and doping-controlled experimental studies seemingly identify lattice-tuned orbital polarization as the underlying physical parameter controlling $T_{\text {IMT }}$ and the Mott gap ${ }^{35}$. Hydrostatic compression ${ }^{39}$ as well as high magnetic field ${ }^{38}$, both accompanied by anomalous elongation along the $c$-axis, weaken the Mott state in CTRO and suggest opportunities to tune the IMT by $c$ - or $b$-axis uniaxial strain. Lastly, this bilayer ruthenate is also remarkable for the inequivalent $b$-axis displacement of its three formula unit $\mathrm{Ca}$ ions ${ }^{40,41}$ that foreseeably impart a polar dipole moment to the Mott insulating phase, rendering hybrid improper ferroelectricity similar to other bilayer perovskites ${ }^{42,43}$. The microscopic consequences of polar domains for the Mott transition have hitherto gone unexplored. Our thorough analysis of domain textures and nano-resolved susceptibilities builds upon previous scanning probe evidence for phase coexistence in $\mathrm{CTRO}^{44}$, and sets the stage for interfacing this insulator-metal transition with the polar degree of freedom.

In this work we directly reveal progression of the strongly firstorder insulator-metal transition in $x=10 \%$ polar CTRO using a cryogenic multi-messenger nano-imaging approach ${ }^{17}$. This method combines application of 10 nanometer-resolved scanning near-field infrared microscopy (nano-IR), which provides a faithful probe of optical properties at deeply sub-wavelength spatial resolutions, with contemporaneous scanning probe sensing modalities like Kelvin probe force microscopy (KPFM), applied here to resolve the work function of domains across the $\mathrm{IMT}^{45}$. Our nano-IR probe is amply suited to distinguish insulator and metal domains emerging across the $\mathrm{IMT}^{46}$ by way of the optical scattering signal $\sigma$ (defined and described in Methods), even at the cryogenic temperatures requisite to study $\mathrm{CRO}^{28,47}$. Moreover, leveraging a nano-resolved protocol denoted scanning nanosusceptibility imaging, we directly reveal the influence of latticeorbital coupling on the insulator-metal transition. Harnessing this approach to tune and visualize Mott nano-textures close to $T_{\text {IMT }}$ enables our direct measurement of an elastic "domain susceptibility", corroborated by our bulk-sensitive elastoresistance probes of the transition and intrinsic to the ordered texture of phase coexistence reported here.

\section{RESULTS}

\section{Mott nano-textures in a bilayer ruthenate}

To enable studying the response of the phase transition to in situ applied strain, individual floating-zone-grown CTRO single crystals of characteristic dimension $100 \times 100 \times 10$ microns $^{3}$ (length $\times$ width $\times$ depth) were glued with their long dimension across the 50-micron gap between the two shear piezoelectric stacks of a home-built uniaxial strain stage (Fig. 1a). With this "strain stage", relative displacements of $\sim \pm 1$ micron can be achieved between the two shear stacks at their top surface with a differential bias of $\pm 500 \mathrm{~V}$ at a temperature of $\mathrm{T} \sim 100 \mathrm{~K}$, thus enabling application of quasi-uniform uniaxial strains exceeding $\pm 2 \%$ to samples of suitable dimension (see Supplementary Methods). For nanoimaging experiments, individual single crystals were cleaved in the (001) plane and pre-mounted with [010] orientation spanning across the gap (Fig. 1b) of our custom strain stage (to within \pm 30 degrees), integrated with an electrically enabled sample holder, and delivered into the ultra-high vacuum cryoSNOM chamber and sample receiver by manipulator arm. Within the sample receiver, electrical contacts were established kinematically to the piezoelectric actuators and to source/drain electrodes across the crystal. Sample temperatures were registered in situ by a diode sensor mounted to the strain stage. Importantly, our imaging measurements address sample regions a distance from the crystal edges much greater than the thickness, assuring local uniformity of the applied strain (details in Supplementary Methods).

We first consider the insulator-metal transition of $10 \% \mathrm{Ti}$ substituted CTRO crystals, with a strongly first-order transition temperature $T_{\mathrm{IMT}} \sim 95 \mathrm{~K}$. Near the transition, nano-IR imaging of large-area (001) crystal facets reveal an emergent striped texture of metal and insulator domains as shown in Fig. 1c, where the nano-IR signal is registered in false color (scale shared with Fig. 1e, f). Figure 1d presents the distribution of metal and insulator regions recorded by nano-IR imaging in the same field of view while cooling the sample through the transition from $T=95.5 \mathrm{~K}$ to $94.5 \mathrm{~K}$, showing at intervals of $\approx 0.3 \mathrm{~K}$ the nucleation and growth of insulating $S$ phase domains in the form of stripes with a characteristic periodicity of several microns perpendicular to the crystallographic $b$-axis. At the phase transition finale, nano-IR reveals extremely narrow metallic $L$ phase domains persisting in an insulating background (Fig. 1e, right panel), whereas the surface topography (left panel) shows linear surface deformation emerging with the larger $L$ phase domain. As indicated in Fig. 1f, the insulating $S$ phase domains produce a nano-IR response characteristically $30 \%$ that of the metallic $L$ phase domains, a value slightly higher than that reported for larger gap Mott insulators $^{28,48}$ but still unambiguously distinguishable from the metal.

To further interrogate sub-micron features associated with phase coexistence, Fig. If presents a multi-messenger nanoimaging examination of a $3 \times 3$ micron striped texture emerging at $\mathrm{T}=95 \mathrm{~K}$. Here we utilize the simultaneous combination of nano-IR imaging, Kelvin probe microscopy, and atomic force microscopy to respectively correlate local metallicity (left panel), work function (center), and surface topography (right) associated with these coexisting striped domains. Figure $1 \mathrm{~g}$ compares the correlative spatial evolution of these properties across the dashed line in the left panel, which over a 4-micron distance intersects several $L$ - and $S$-phase domains. The nano-IR response $\sigma$ (red curve) reveals interphase boundaries sharp on the scale of $200 \mathrm{~nm}$. However, the local work function (orange curve), registered by KPFM as the "contact potential difference" $\Delta \Phi=\Phi_{\text {CTRO }}-\Phi_{\text {tip }}$ between work functions $\Phi$ of the sample and the nano-IR probe (Methods), consistently anti-correlates in real-space with local metallicity. We detect an increase in work function $\Delta \Phi_{\text {CTRO }}=\Phi_{L}-\Phi_{S}$ from the metallic $L$ to the insulating $S$ phase of about $20 \mathrm{mV}$, consistent in magnitude with opening of the latter's semiconducting gap ${ }^{35}$ and similar to the change in work function through the insulator-metal transition of an analogous correlated oxide, $\mathrm{VO}_{2}{ }^{49}$, thus affirming our assignment of insulator and metal domains based on infrared contrast. The central panel of Fig. If shows spatial variations in $\Delta \Phi$ of order $5 \mathrm{mV}$ at characteristic length scales of $100 \mathrm{~nm}$, comparable to the spatial resolution of our KPFM technique, indicating extreme sensitivity to variations presumably in surface oxidation of the crystal facet, cleaved ex-situ under ambient conditions prior to attachment onto our strain stage.

Work function variations (details we defer to forthcoming work) show no noticeable association with the local surface topography (Fig. 1f, right), which is atomically smooth to the precision of our AFM, with exception of notable buckling in the crystal surface on the scale of several nanometers associated directly with the underlying $L$ and $S$ phase domains and only observed during their coexistence; the bottom panel of Fig. $1 \mathrm{~g}$ highlights this association. While similar to observations in phase-change oxides like $\mathrm{VO}_{2}$ and $\mathrm{V}_{2} \mathrm{O}_{3}$ thin films ${ }^{28,48}$, this buckling pattern signifies the underlying domain structure of coexisting $L$ and $S$ phases into the volume of this ruthenate single crystal ${ }^{50}$. In particular, we expect the nearly $1 \%$ expansion in $c$-axis lattice constant through 
b

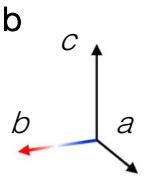

C
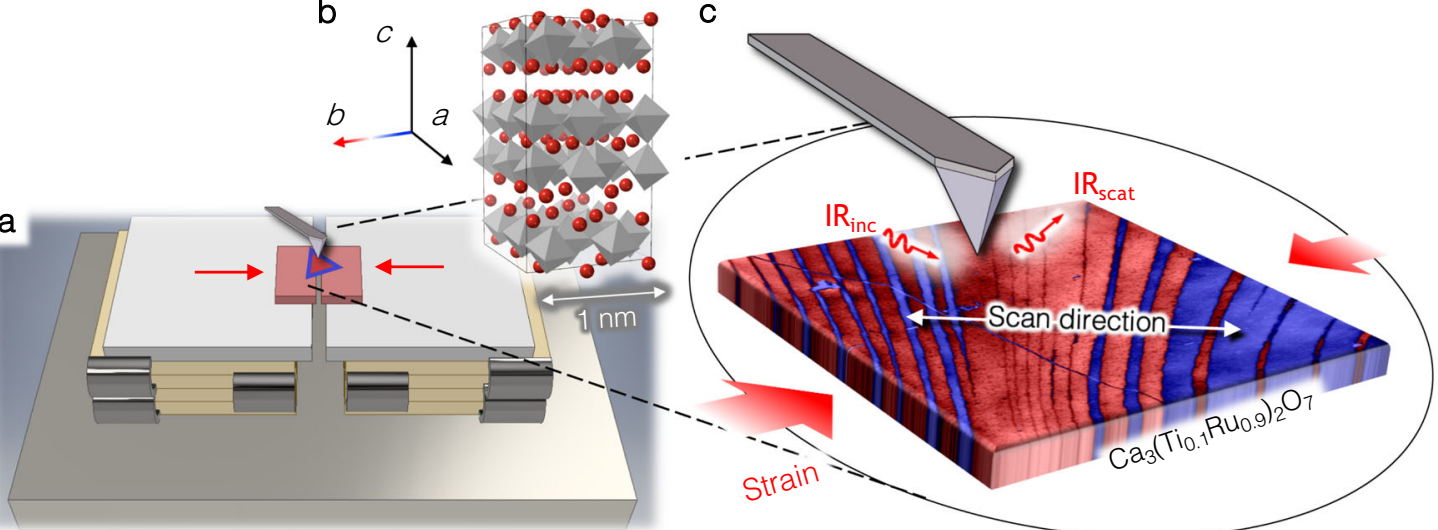

cooling
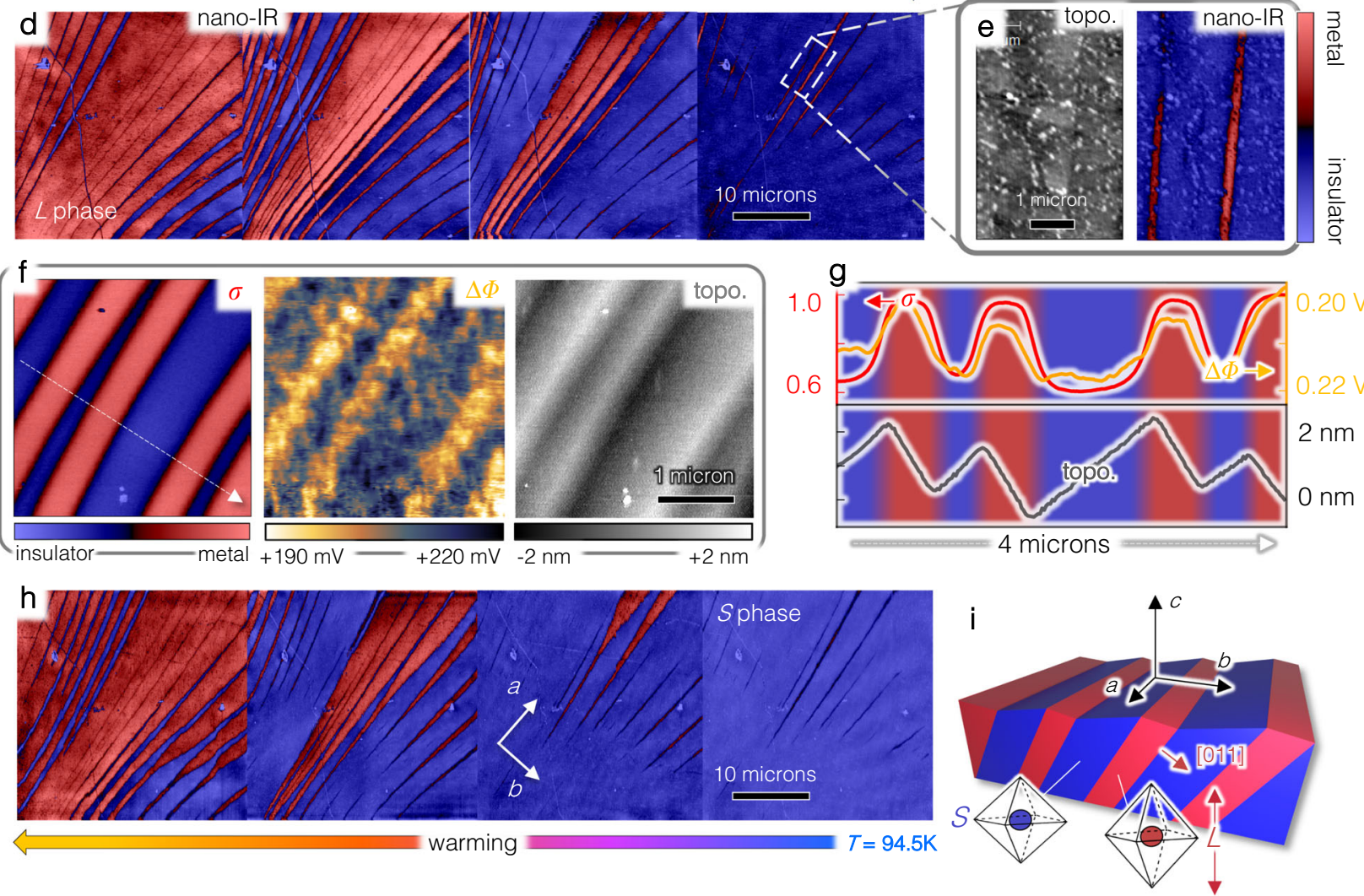

Fig. 1 Mott nano-textures in a bilayer ruthenate. a Schematic depiction of a 100 micron-scale thin $\mathrm{Ca}_{3}\left(\mathrm{Ti}_{0.1} \mathrm{Ru}_{0.9}\right)_{2} \mathrm{O}_{7} \mathrm{crystal}$ mounted to a home-built strain stage inside the low-temperature nanoscope. b Crystal structure of the bilayer ruthenate; our experiments apply uniaxial strain within 30 degrees of the crystallographic b-axis. c Metal/insulator nano-textures are detected by nano-imaging the crystal surface (abplane) at $100 \mathrm{meV}$ probing energy during in situ application of strain. d Striped nanotextures emerging while cooling the crystal from the metallic $L$ phase through the Mott transition from $T=95.5 \mathrm{~K}$ to $94.5 \mathrm{~K}$ without applied external strain; images shown were acquired at $\approx 0.3 \mathrm{~K}$ intervals. e Minority phases take the form of acicular domains; topography shares color scale with $\mathbf{f}$. $\mathbf{f}$ Correlative topography, contact potential difference $\Delta \Phi$ (see text) and nano-IR signal $\sigma$ recorded across of a series of $L$ phase domains; the color-scale for $\sigma$ indicates the nano-IR response from the insulating phase is $30 \%$ that of the metal. $\mathbf{g}$ Correlative line-profiles along the arrow in (f) associated with each imaging channel; the surface work function anticorrelates local metallicity, and expansion of $L$ domains buckles the surface topography. $\mathbf{h}$ Same region as (d) imaged while warming from the insulating $L$ phase; images shown were acquired at $\approx 0.3 \mathrm{~K}$ intervals. i Inferred schematic structure of coexisting $L$ and $S$ domains ([011] habit plane) showing associated $\mathrm{RuO}_{6}$ octahedral deformations.

the insulator-metal transition ${ }^{31}$ to dominate buckling of the (001) surface, whereas the accompanying $>1 \% \quad b$-axis contraction should influence the preferred orientation of inter-phase domain boundaries ${ }^{51}$. As shown schematically in Fig. 1i, detailed considerations of elastic energy minimization (Supplementary Methods) in this case predict inter-phase domains oriented nearly

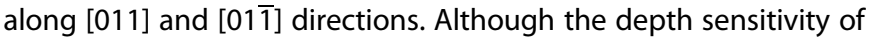

our nano-IR probe $\left(\sim 50 \mathrm{~nm}^{52}\right)$ is too limited to directly distinguish these possibilities, the latter orientation is qualitatively consistent with the observed "angled" buckling pattern with topographic peaks and troughs aligned along $L-S$ and $S-L$ inter-phase boundaries, respectively (Fig. $1 \mathrm{~g}$ ). Figure $1 \mathrm{~h}$ presents the same field of view as Fig. 1d imaged while warming the sample back to $95.5 \mathrm{~K}$, where the reverse scenario of nucleation and growth of 

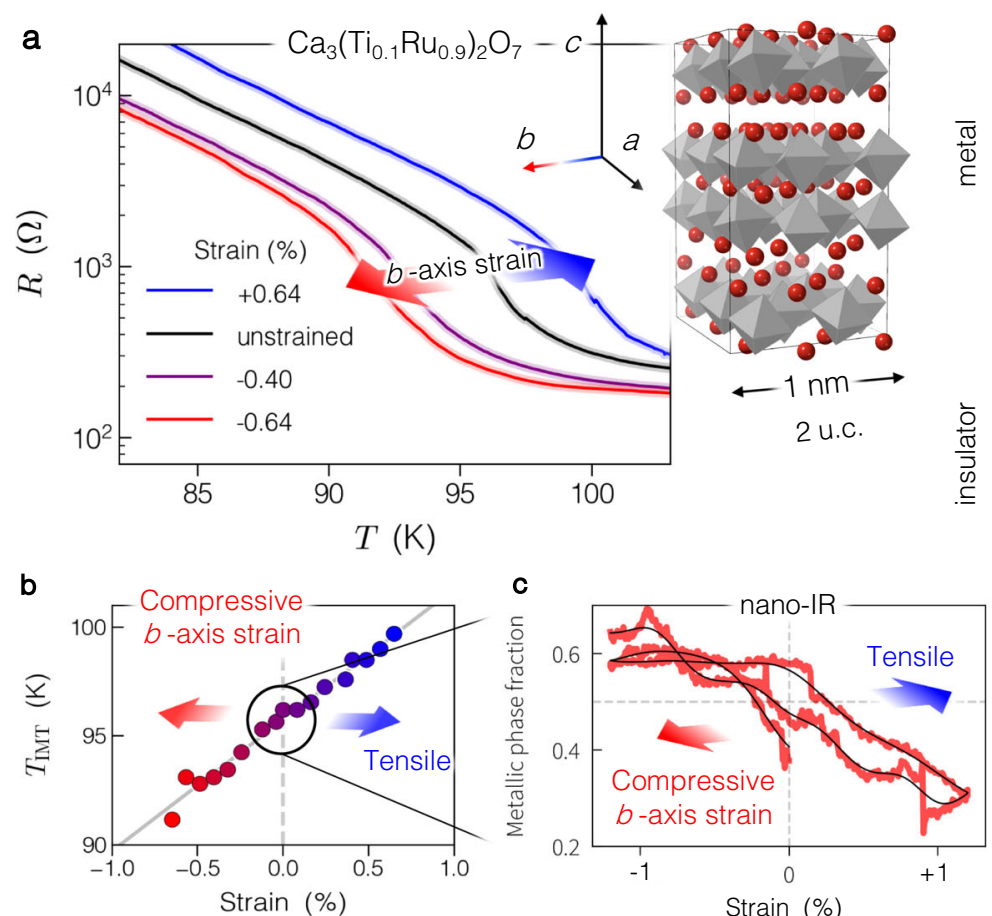

d

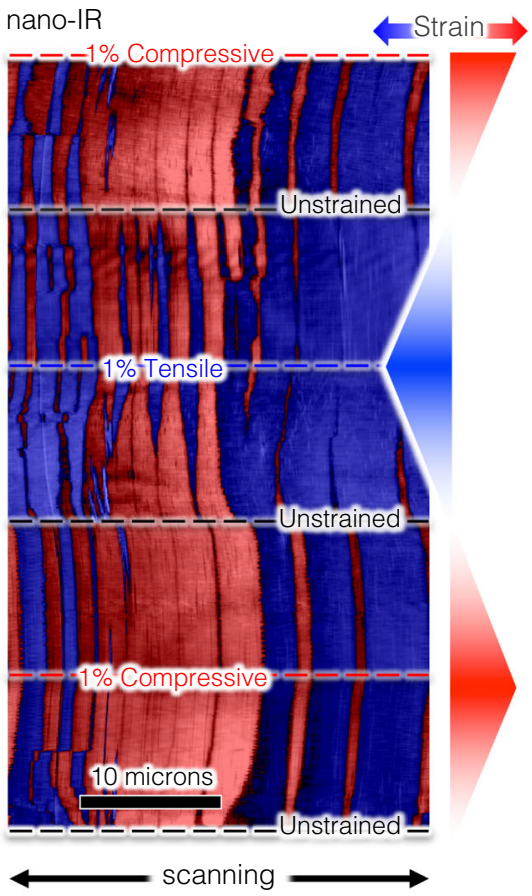

Fig. 2 Strain modulation of the Mott transition. a Two-probe electrical resistance of the crystal in Fig. 1 across the insulator-metal transition under compressive $(\varepsilon<0)$ and tensile $(\varepsilon>0) b$-axis strain conditions; the overall resistive transition shifts in temperature. Crystallographic axes are labeled on eight rendered unit cells of the crystal structure. $\mathbf{b}$ Insulator-metal transition temperature $\mathrm{T}_{\mathrm{MIT}}$ (identified by a peak in $R^{-1} d R / d T$ ) identified versus applied $b$-axis strain. c Areal metallic phase fraction at $T=95 \mathrm{~K}$ identified by repeatedly nano-imaging along the line identified in Fig. 1c versus in situ applied strain; phase fractions can be modulated by $40 \%$ under a $2 \%$ variation in strain. Small arrows indicate the strain sweep sequence. d Spatially resolved insulator-metal nano-texture along the same scan line quantified in (c) versus dynamically tuned strain; beginning from an unstrained state (bottom) metallic domains enlarge under $\varepsilon=-1 \%$ compressive strain, then diminish under $\varepsilon=+1 \%$ tensile strain, and can be reversibly restored under renewed compressive strain.

$L$ phase domains proceeds through the insulator-metal transition. The similarity of metal and insulator phase fractions observed at comparable temperatures during cooling and warming (Fig. 1d, h, respectively) reveals a local hysteresis of less than $0.5 \mathrm{~K}$, which is comparable to the abruptness of the observed transition in the area of the crystal investigated here. Nevertheless, we note the phase transition proceeds rather non-uniformly across other regions of this crystal addressable by our probe, spanning a temperature range of at least $5 \mathrm{~K}$, consistent with further characterizations that we discuss next. This spatially inhomogeneous transition owes perhaps to a non-uniform distribution of "built-in" strain owing to the triangular shape of this particular crystal clamped to our strain device. Overall, these introductory characterizations reveal that (i) nano-IR and KPFM imaging are faithful correlative probes of the low-temperature CTRO insulatormetal transition, (ii) the micron-scale periodicities of striped domain textures indicate dominance of elastic energetics between incommensurate $L$ and $S$ structural phases, and (iii) our nanoimaging probes register a two-dimensional cross-section of an underlying three-dimensional domain texture forming spontaneously throughout the crystal volume during the IMT.

\section{Scanning nano-susceptibility imaging of strain-tuned Mott domains}

Our observations of striped domains showcase the influence of long-range strain fields on the spatial texture of insulator-metal phase coexistence, prompting our application of external uniaxial strain to manipulate these phase textures in situ. We begin by registering the resistive transition of the triangular single microcrystal mounted to our strain stage at fixed levels of uniaxial strain aligned nearly along the crystallographic b-axis. Figure $2 a$ presents thermal sweeps probing the resistive transition of the entire crystal volume, showing a full transition spanning about $5 \mathrm{~K}$. Application of exemplary tensile (positive) and compressive (negative) $b$-axis strains reveals an overall shift of the resistance curve by several degrees $\mathrm{K}$ under less than half a percent strain application, accompanied by an overall increase (decrease) of the insulating state resistance that we ascribe to possible changes in the insulating gap. Defining a "characteristic" transition temperature $T_{\text {IMT }}$ by the onset of a sharp upturn in sample resistance (viz. maximum in $R^{-1} \partial R / \partial T$ ) entering the insulating state, Fig. $1 \mathrm{~b}$ summarizes elastic susceptibility of the resistive transition. Whereas $T_{\mathrm{IMT}} \approx 95 \mathrm{~K}$ for the unstrained crystal, modest application of $0.5 \%$ tensile strain (temperature-dependent calibration of the strain stage detailed in $\mathrm{SI}$ ) shifts the transition temperature by $+2.5 \mathrm{~K}$, whereas $-0.5 \%$ compressive strain suppresses the transition by $-2.5 \mathrm{~K}$, signifying an enormous uniaxial $b$-axis elastic susceptibility of $\frac{\partial T_{\text {IMT }}}{\partial \varepsilon}=5 \times 10^{2} \mathrm{~K}(\varepsilon$ hereafter denotes the $b$-axis dilatory component of the $2^{\text {nd }}$ rank strain tensor $\underline{\varepsilon}$ ). This finding accords with previous trends resolved across a series of doping concentrations ${ }^{35}$, but here resolved in a single CTRO crystal. Qualitatively, we envision that (i) compressive stabilization of the reduced- $b$ axis $L$ phase and (ii) tensile stabilization of the enlarged$b$ axis $S$ phase together form an underlying mechanism for this susceptibility. To test this hypothesis, we performed in situ nano-IR imaging of the same microcrystal at $T=95 \mathrm{~K}$ under comparable strain conditions. By continuously scanning perpendicular to the insulator-metal stripes (parallel to the $b$-axis, indicated in Fig. 1c), monitoring the nano-IR signal $\sigma$, and simultaneously sweeping the level of uniaxial strain delivered to the crystal, we directly resolved the real-space impact on insulator and metal phases. Figure $2 \mathrm{~d}$ reveals the 1-dimensional lateral distribution (along the horizontal spatial axis) of insulator-metal domains from an initially unstrained configuration (bottom), and continuing first to a $1 \%$ compressively 

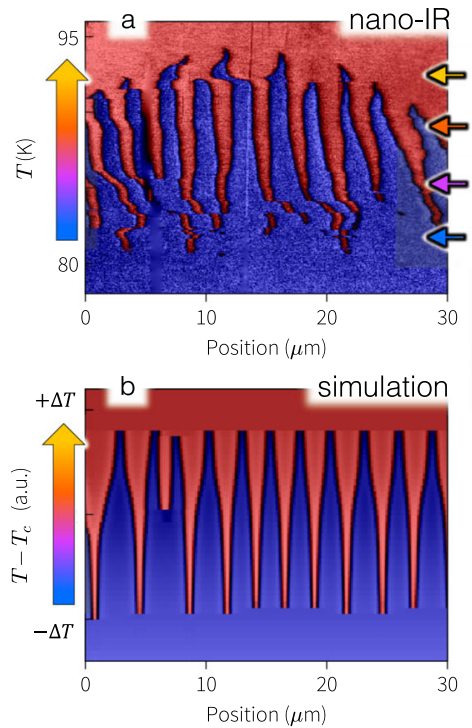

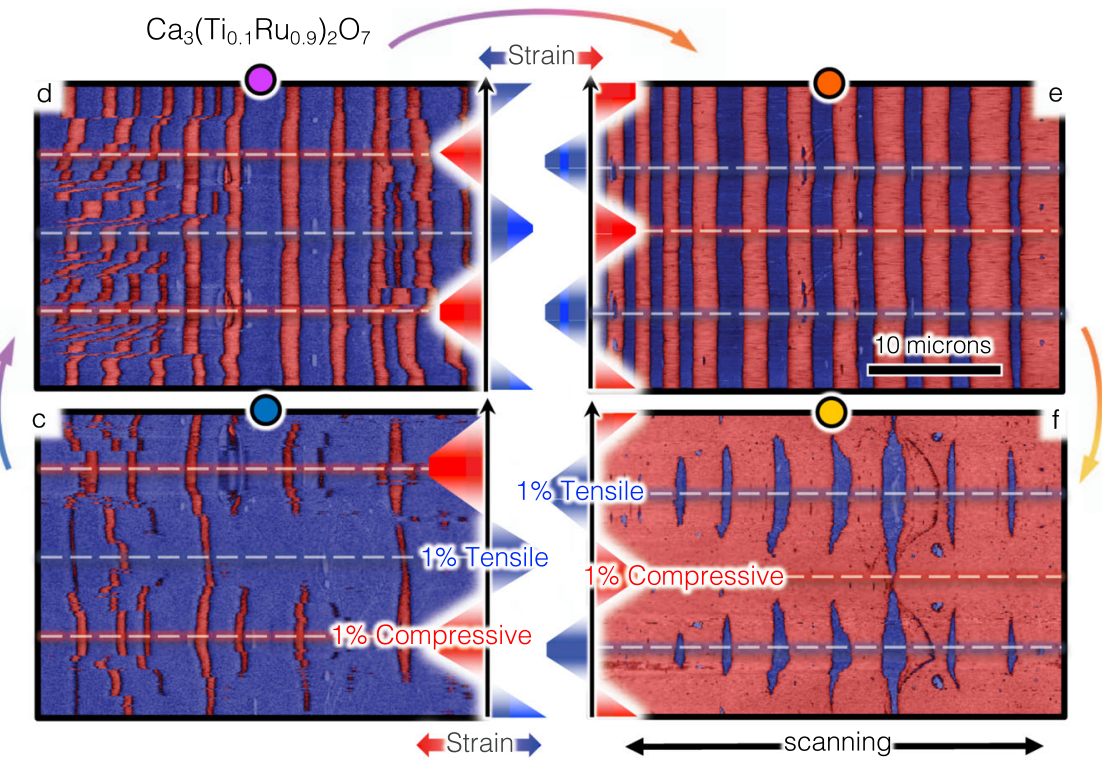

Fig. 3 Scanning nano-susceptibility of temperature- and strain-tuned Mott domains. a Repeated nano-imaging along a fixed-line parallel to the $b$-axis of a 300 micron-long rectangular crystal while linearly warming the sample temperature resolves thermal evolution of domain textures. b A phase-field simulation of a strain-coupled transition (see text) replicates the observed domain evolution. c-f Repeated nanoimaging along the same line as (a) but at fixed temperatures, identified by colored circles associated with arrows in (a), and under programmable strain-sweeps. c At low temperature compressive strain nucleates metallic stripes; $\mathbf{f}$ at high temperature tensile strain nucleates insulating stripes; d, e intermediate temperatures alternatively reveal rigidity or complex rearrangement of domain textures.

strained state where we resolve outward growth of preexisting metallic $S$ phase stripes. Next, application of $1 \%$ tensile strain reduces the areal fraction of metal, yielding a majority insulating state where several metallic domains have vanished completely. This protocol of "scanning nano-susceptibility imaging" (SNSImaging) is entirely reversible, as demonstrated by the concluding (top) majority metallic configuration replicated in both domain positions and shapes at $1 \%$ compressive strain.

Note that this strain application is accompanied by an overall leftward (rightward) shift of the scanned field of view due to physical motion of the crystal during compressive (tensile) actuation of the strain stage. These data are dominated by the growth and reduction of pre-nucleated metallic domains, rather than nucleation of new domains. Thus, since our scan perpendicular to the orientation of these stripes provides a faithful view of their overall size, we can quantify the elastic susceptibility of these phases by recording the metallic phase fraction versus strain after excluding the contribution of metallic domains that enter or leave the field of view. Figure $2 c$ presents the metallic phase fraction $f$ extracted in this fashion versus strain, revealing a roughly linear dimensionless elastic susceptibility $X_{\text {elas }} \equiv \frac{\partial f}{\partial \varepsilon}$ of at least 15 , suggesting the possibility to completely modulate an isothermal Mott transition in CTRO at a $b$-axis strain of $\varepsilon \leq 15^{-1} \approx 7 \%$. While this would presumably exceed onset of substantial nonlinearities or even the damage threshold for our 10 microns-thick single crystals (and the range of the strain stage used in this study), it is consistent with the typical strains demonstrated to modulate the Mott transition in thin films of the related ruthenate $\mathrm{Ca}_{2} \mathrm{RuO}_{4}{ }^{13,14,53}$ and is foreseeably accessible without damage in thin perovskite membranes ${ }^{54}$. Given the apparent orientation of stripe domains in this crystal (viz. Fig. 1d) and evident misalignment of the $b$-axis by as much as $30^{\circ}$ relative to our applied strain, these observations demonstrate a robust strain susceptibility. This owes perhaps to vanishing strain susceptibility along the crystal $a$ axis, which we might anticipate from the reported constancy of the $a$-axis across the insulator-metal transition for $\mathrm{Ca}_{3} \mathrm{Ru}_{2} \mathrm{O}_{7}$ crystals with substitution levels of $3 \mathrm{~d}$ metals similar to our own ${ }^{35}$.
Notably, we associate the minimal strain hysteresis presented in Fig. 2c solely with the memory effect of piezoelectric actuators used on our stage, suggesting surprisingly prompt responsivity of the striped insulator-metal microtexture to applied strain. To interrogate this pliancy in greater detail, we conducted SNSImaging on a second crystal with a more ideal 300 micron-long rectangular shape and 10-micron thickness presenting a uniform cross-section across the gap of our strain stage. This crystal was found to present a more spatially uniform quasi-1-dimensional "domain lattice" texture at several fixed temperatures through the Mott transition. Figure 3a presents thermal SNSImaging recorded parallel to the crystal $b$-axis while linearly ramping the sample temperature from 80 to $95 \mathrm{~K}$ under zero applied strain, revealing initial nucleation of $\mathrm{S}$-phase stripes that settle into a quasi-1dimensional lattice texture with uniform $\sim 2 \mu \mathrm{m}$ periodicity, followed by a relatively abrupt merger of metallic domains into a uniform state at $T \approx 93 \mathrm{~K}$. We next cycle the sample temperature, and warm again to fixed temperatures indicated in Fig. 3a by colored arrows whereupon we perform strain-modulated SNSImaging. These temperatures are chosen to stabilize distinct initial configurations of the domain lattice to register $X_{\text {elas }}$ at (unstrained) metallic phase fractions of $10 \%, 30 \%, 60 \%$, and $90 \%$ associated with Fig. 3c-f, where colored dots correspond to temperatures identified by the colored arrows. At the onset of the IMT, Fig. 3c records SNSImaging where ramping from an initially unstrained configuration up to $+1 \%$ tensile strain yields only a few prenucleated metallic domains, but after strain removal followed by application of $-1 \%$ compressive strain we observe nucleation and growth of 4-5 additional metallic domains that position spontaneously to establish the characteristic lattice periodicity. This response is reversible across the subsequent strain sweeps indicated by adjacent blue (tensile) and red (compressive) colored shading. This regime of strain-modulated phase coexistence is plainly dominated by induced nucleation, whereas Fig. 3d presents a quite different response at $30 \%$ metallic fraction. Here preexisting metallic domains undergo a complex "dance" of mergers (splitting) under compressive (tensile) strain as a means to grow (shrink) the metallic phase fraction. These events are 

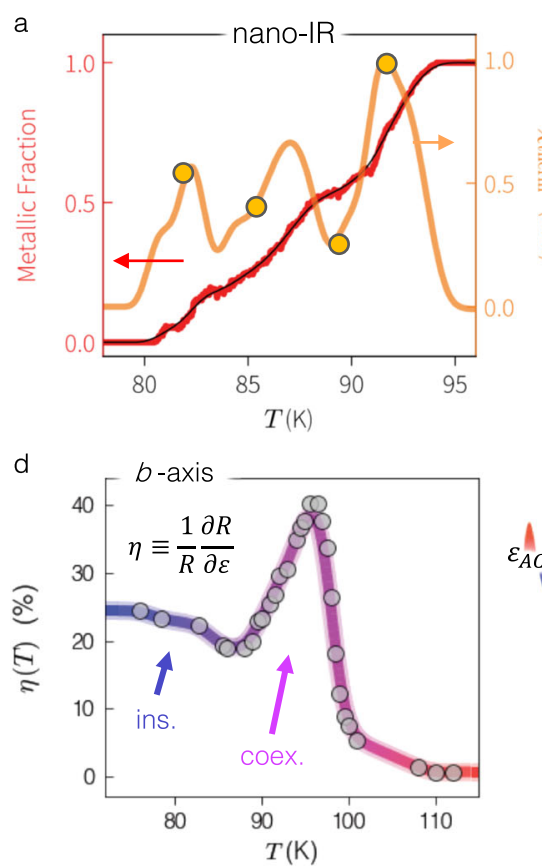

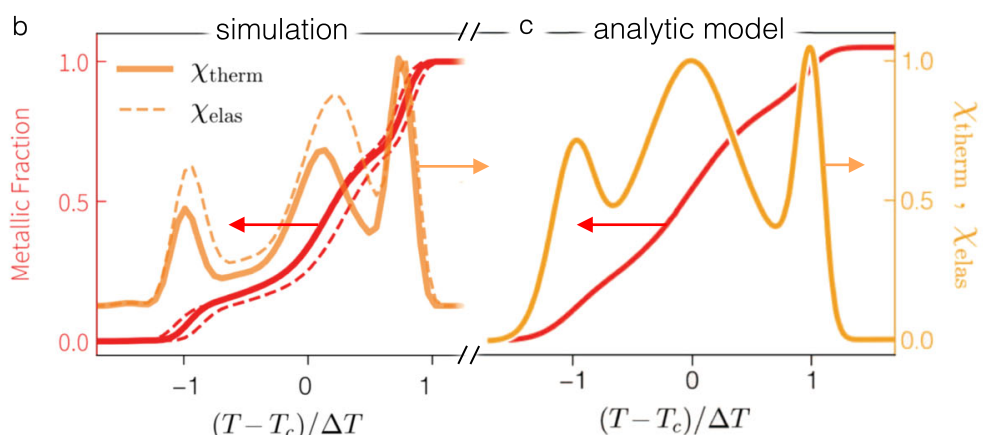

$\left(T-T_{c}\right) / \Delta T$

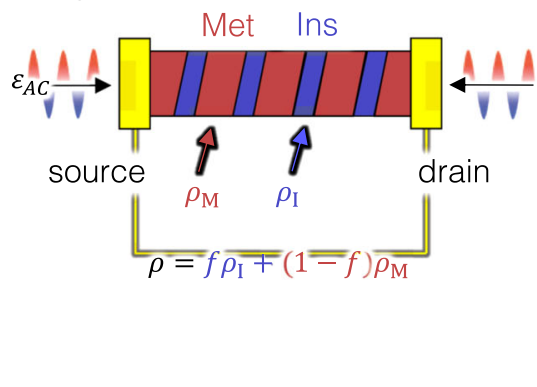

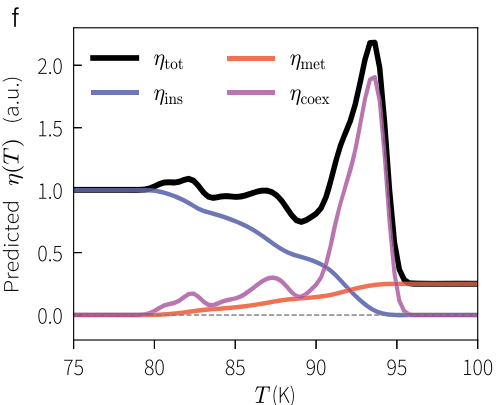

Fig. 4 Signatures of emergent Mott domain susceptibility. a T-dependent metallic fraction $f$ from Fig. 3a together with thermal susceptibility $X_{\text {therm }}=\mathrm{d} f / \mathrm{d} T$ (orange); circular points quantify the elastic susceptibility $X_{\text {elas }}=\mathrm{d} f / \mathrm{d} \varepsilon$ identified at similar temperatures from Fig. 3c-f. b Phase-field simulations and (c) a semi-analytic model of domain evolution both predict the characteristic temperature dependence of $X_{\text {therm }}$ and $X_{\text {elas }}$. $\mathbf{d}$ An anomalous peak in elastoresistance $\eta$ emerges in the phase coexistence regime of the thermal transition; gray markers indicate experimental data whereas the colored curve is a guide to the eye; ins. and coex. respectively indicate insulating and phasecoexistent temperature regimes. e A simple circuit model allows to (f) predict $\eta$ based on the elastic susceptibility inferred from (a) for the same insulator-metal mixture observed by nano-IR imaging (see text).

accompanied by a positional shift of adjacent stripes by a single lattice spacing seemingly to maintain integrity of the domain lattice and prevent dislocations, even as the "natural" lattice periodicity itself appears notably strain-dependent, e.g. in Fig. 3d dropping from $\sim 3$ to 2 microns while sweeping from tensile to compressive strain. Meanwhile, at higher initial unstrained metallic phase fraction $(60 \%$, Fig. 1e), the domain lattice becomes notably "stiff" and comparatively insensitive to our application of external strain, neither shifting nor appreciably growing existing domains. Lastly, Fig. If presents the domain susceptibility from a dominantly metallic configuration (in the identical field of view) near the conclusion of the IMT, where we find comparatively large elastic response capable of both nucleating and growing insulating domains to an areal fraction exceeding $20 \%$.

\section{Nano-resolved elastic domain susceptibility}

Our SNSImaging results systematically demonstrate a detailed non-uniformity in elastic susceptibility of the domain lattice versus temperature, as summarized in Fig. 4a. Here we superimpose the unstrained temperature-modulated metallic phase fraction $f(T)$ (red curve, extracted from the same sample region in Fig. 3a) with its associated thermal susceptibility (viz. $X_{\text {therm }} \equiv \frac{\partial f}{\partial T}$, orange curve, smoothed); we scale the latter to its maximum value. Instead of a constant ramp or sigmoidal progression, $f(T)$ presents a series of three "jumps" separated by valleys of comparatively reduced susceptibility; this behavior manifests as three peaks in $X_{\text {therm }}$ at $f \approx 15,40$, and $85 \%$. Such three-fold progression in $f(T)$ was verified by SNSImaging at two other sample regions also presenting a near-uniform domain lattice (not shown). Moreover, when we add distinct data points (orange markers) reflecting the variable $X_{\text {elas }}$ extracted from data in Fig. 1c-f $\left(X_{\text {elas }} \propto \frac{\Delta f}{\Delta \varepsilon}\right.$ with constant $\Delta \varepsilon=2 \%$; scaled again to highest $X_{\text {elas }}$ ), we find intriguing qualitative agreement with these systematic variations in $X_{\text {therm }}$. We suppose these non-monotonic trends in susceptibility are nontrivially tied to the elastic energy "landscape" which also dictates the quasi-equilibrium configuration of the domain microtexture.

We employ a simplified Landau theory of the strain-coupled IMT to reveal the essential physics underlying these energetics. Denoting by $\psi$ the amplitude of the coelastic distortion describing the insulating $S$-phase $(\psi>0$, meanwhile denoting the $L$-phase by $\psi=0$ ) we can approximate the Landau free energy density $F$ of this first-order insulator-metal transition to quartic order in $\psi$ by:

$F \approx \frac{1}{2} K|\nabla \psi|^{2}+a\left(T-T_{\text {IMT }}^{0}\right) \psi^{2}-\beta \psi^{3}+\gamma \psi^{4}-\delta \varepsilon K\left(\varepsilon_{\text {ext }}+\varepsilon_{\text {int }}\right) \psi$.

Here $\kappa$ denotes a stiffness energy per unit area of insulatormetal "domain boundaries", $T_{\text {IMT }}^{0}$ denotes the "unstrained" IMT temperature, and quantities $\alpha, \beta$, and $\gamma$ are energetic constants. Meanwhile, $\delta \varepsilon$ is the $b$-axis dilatory component of the $2^{\text {nd }}$ rank spontaneous strain tensor $(\delta \underline{\varepsilon}$, nonzero only for $c$ - and $b$-axis components), $K$ is a $b$-axis elastic stiffness constant of the crystal, and we have split the $b$-axis uniaxial strain $\varepsilon=\varepsilon_{\text {ext }}+\varepsilon_{\text {int }}$ into a spatially homogeneous external part $\varepsilon_{\text {ext }}$ (viz. applied by our strain stage) and an internal inhomogeneous part $\varepsilon_{\text {int }}$ arising from accommodation strain between coexistent $S$ - and $L$-domains. The inhomogeneous strain is determined self consistently from the real-space domain distribution of $\psi$ (simulation details provided in ref. $\left.{ }^{17}\right)$. By properly encapsulating the essential competition of domain wall and elastic accommodation energies, similar Landau models have shown success in describing nano-imaging results of periodic domain textures in a variety of strain-coupled first-order transitions of complex oxide thin films ${ }^{17,47}$ as well as single crystals ${ }^{27}$, including the related $\mathrm{Ca}_{2} \mathrm{RuO}_{4}{ }^{50}$. In particular, the interplay of $\kappa$ and $a$ determines the width $I_{D W}$ of domains walls, less than $100 \mathrm{~nm}$ according to our observations (Fig. 1g), whereas the microns-scale periodicity $\lambda$ of insulator-metal stripes follows essentially from $K$ and $K$. Accordingly, we first used Eq. (1) to 
describe a surficial "slab" of our crystal undergoing the IMT without external strain $\left(\varepsilon_{\text {ext }}=0\right)$, and in a periodic area of the crystal $a b$-plane we numerically minimized the integrated free energy to obtain real-space evolution of $\psi$ versus temperature (free parameters were chosen only to establish the observed domain lattice periodicity; see refs. ${ }^{17,50}$ ). Figure $3 b$ presents $\psi$ in false color (identifying insulator and metal) across a line parallel to the simulation $b$-axis (2D maps at fixed $T$ presented in $\mathrm{SI}$ ), demonstrating nucleation, growth, and evolution of the obtained quasi-1-dimensional domain lattice across the IMT; here $T_{c}$ denotes the temperature at which $L$ and $S$ become equal-energy phases and the range $\Delta T$ spans their metastability.

Figure $4 \mathrm{~b}$ presents the computed $T$-dependent metallic phase fraction (red) and simulated thermal susceptibility (orange) for comparison against our measurements in Fig. 4a, showing a remarkable reproduction of the observed three-fold thermal susceptibility. Although quadratic coupling of $\psi$ to $T$ is technically distinct from its linear coupling to $\varepsilon_{\text {ext }}$ as predicted by this coelastic theory, we nevertheless regard $\varepsilon_{\text {ext }}$ as a "forcing" term capable of modulating the temperature $T_{\mathrm{IMT}}$ below which the $L$-phase destabilizes, in accord with Fig. 2a. Therefore, repeating the same simulation with $\varepsilon_{\text {ext }}=+1 \%$ and $-1 \%$ further suppresses or stabilizes the metallic phase according to expectation, rendering new phase fractions $f^{+}(T)$ and $f^{-}(T)$ (lower and upper dashed red curves of Fig. 4b). Their difference provides a measure $X_{\text {elas }} \propto\left(f^{-}-f^{+}\right) / \Delta \varepsilon$ of the elastic domain susceptibility (dashed orange curve), also bearing an uncanny resemblance to the threefold structure of $X_{\text {therm }}$. We can rationalize the resemblance of $X$ elas,therm under examination of Eq. (1), where the effect of $\varepsilon_{\text {ext }}<0$ $(>0)$ is to energetically (dis)favor the metallic phase. A mean-field treatment quantifies the associated shift transition temperature as $T_{\mathrm{IMT}}=T_{\text {IMT }}^{0} \mp \delta T$, with $\delta T \sim \delta \varepsilon K \varepsilon_{\text {ext }} / \psi$ and $\psi$ our order parameter's areal average.

Though our numerical results affirm that Eq. (1) accounts for the physics underlying unexpected structures observed in these susceptibilities, further explanation demands an analytic treatment of the elastic energy landscape. Recognizing the quasi-1dimensional behavior of phase separation here reported in this crystal, we employ a variational minimization to a simplified 1dimensional form of Eq. (1) (see Methods and Supplementary Discussion) to explore the analytically deduced $T$-dependent phase fraction and thermal susceptibility presented in Fig. 4c. In this model, susceptibility peaks near $f \approx 0,1$ associate first with the energy barrier of forming $L$ - or S-phase stripes with a minimum size exceeding $I_{D W}$, followed abruptly by energetically favored formation of a coherent $\lambda$-periodic stripe lattice (Supplementary Discussion). A spatially inhomogeneous energy landscape (viz. $T_{1 \mathrm{MT}}^{0}$ ) suffices to broaden $X_{\text {elas.therm }}$ associated with this abrupt transition. On the other hand, the central susceptibility peak originates from a strong predisposition of the domain lattice towards $f \sim 1 / 2$, where strain fields of mismatching but energetically equal surficial $L$ - and $S$-phase domains can be best accommodated by the bulk of the crystal at minimal elastic distortion. The central susceptibility peak affords therefore a "sensor" for the $f \sim 1 / 2$ condition of equal-energy phases forming a symmetric stripe lattice. Moreover, we propose the detailed shape of this peak in Fig. 4a, b could provide experimental access to the T-dependent electronic energy difference $\Delta E_{L, S}(T)$ between competing metastable phases. In particular, the symmetry of the susceptibility peaks observed in Fig. $4 a$ with respect to the midpoint temperature of the transition (where equal metallic and insulating phase fractions emerge) may suggest an underlying linear $\psi-T$ coupling in common with the simple model treated in Fig. 4c, and also theoretically motivated for the related Mott insulator $\mathrm{Ca}_{2} \mathrm{RuO}_{4}{ }^{14}$. This feature differs noticeably from the asymmetric peak in Fig. 4b following from the "conventional" double-well energy (Eq. (1)) assumed by many first-order Landau theories. However, we note the experimentally inferred (Fig. 1) but indirectly resolved three-dimensional structure of domains comprising the lattice texture may also play a detailed role.

\section{DISCUSSION}

Our findings by scanning nano-susceptibility imaging demonstrate that, while external strain can deterministically modulate the macroscopic Mott transition in this prototypic 4d-metal oxide, implicit accommodation strain of coexisting phases plays an equally important role for governing both (i) their real-space texture and (ii) the elastic response of the resulting domain lattice - a property we denote "domain susceptibility." In fact, even the macroscopic susceptibility of this Mott insulator to uniaxial strain shows hallmarks of these phenomena. Figure $4 \mathrm{~d}$ presents bulk elastoresistance measurements along the $b$-axis of a CTRO crystal similar to that explored in Figs. 3 and $4 a$ (experimental details in Methods); here the elastoresistance $\eta \equiv R^{-1} \partial R / \partial \varepsilon$ records relative changes in crystal resistance under uniaxial strain, a metric previously applied to measure "divergent" nematic susceptibilities across continuous phase transitions ${ }^{18}$. In that context, an observed peak in $\eta$ at $T \approx 95 \mathrm{~K}$ through our first-order transition may come as a surprise until reconciled with our SNSImaging results. Envisioning our phase-coexistent crystal as a quasi-onedimensional network of metal and insulator domains (Fig. 4e) with respective resistivities $\rho_{M, 1}$, the overall $b$-axis resistivity $\rho=$ $f \rho_{l}-(1-f) \rho_{M}$ will expectedly show a response $\rho_{A C}$ to oscillating uniaxial strain $\varepsilon_{A C}$ related to $\eta$ by:

$$
\begin{gathered}
\rho \eta=\frac{\rho_{A C}}{\varepsilon_{A C}} \propto X_{\text {elas }}\left(\rho_{l}-\rho_{M}\right)+f \partial_{\varepsilon} \rho_{l}+(1-f) \partial_{\varepsilon} \rho_{M} \\
\therefore \eta \approx \eta_{\text {coex }}+f \eta_{l}+(1-f) \eta_{M} .
\end{gathered}
$$

The second equation follows in our case where $\rho_{l} \gg \rho_{M}$, with elastoresistances for the end-phase defined by $\eta_{I, M} \equiv \rho_{I, M}^{-1} \partial_{\varepsilon} \rho_{I, M}$. Crucially, this framework predicts an emergent positive elastoresistence $\eta_{\text {coex }}$ associated solely with the onset of phase coexistence and its consequent elastic domain susceptibility given by $\eta_{\text {coex }} \equiv X_{\text {elas }} / f$. To generate a simple qualitative model for the data recorded in Fig. 4d, we apply our inferences for $f(T)$ and $X_{\text {elas }}(T)$ from Fig. $4 a$, together with $\eta_{I, M}$ discernible from the end-phase values of $\eta$, resulting in the characteristic line-shape shown in Fig. 4f. This model predicts an obvious asymmetric peak in elastoresistance upon formation of the stripe domain lattice below $T=95 \mathrm{~K}$, in remarkable accord with our measurements of a similar crystal in Fig. 4d. The agreement only falters in the thermal width of the elastoresistance peak; however, this is easily reconciled by considering that our nano-imaging results in Fig. 3 resolve only a narrow portion of the crystal, whereas $T_{\text {IMT }}$ may vary throughout the crystal by $5 \mathrm{~K}$ or more, easily accounting for the comparative thermal breadth of the elastoresistive response. Therefore, these findings demonstrate elastoresistance as a useful macroscopic reporter of phase coexistence across first-order transitions in which the constituent electronic phases are coupled to strain, as in the Mott insulator $\mathrm{Ca}_{3}\left(\mathrm{Ti}_{\mathrm{x}} \mathrm{Ru}_{1-\mathrm{x}}\right)_{2} \mathrm{O}_{7}$. Moreover, we interpret the absence of any detectible peak in $a$-axis elastoresistance measured across this insulator-metal transition with the vanishing elastic susceptibility of the stripe domain lattice parallel to the underlying $L$ - and $S$-phase domains. We, therefore, envision orientation-resolved elastoresistance as a promising probe of domain morphologies amidst phase coexistence in systems (and internal volumes) not otherwise directly addressable by nanoprobes like SNSImaging. These correlative techniques thus provide useful complementarity.

We have demonstrated that the Mott insulator-transition in single crystals of CTRO provides an uncommonly pristine platform to resolve the intrinsic response of self-organized domain textures to external stimuli like strain and temperature. Complemented by bulk uniaxial elastoresistance measurements, our low-temperature scanning nano-susceptibility imaging provides real-space access 
to strain-tuned domain textures evolving across the insulatormetal transition of this Mott crystal. Our nano-scale elastic probe should prove versatile for tracking phase-change susceptibilities with unprecedented spatial resolution in other correlated $4 \mathrm{~d}$ and $5 \mathrm{~d}$ transition metal oxides, and in correlated electron solids more broadly. For instance, the yet-mysterious electrical switching characteristics common to both $\mathrm{Sr}_{2} \mathrm{RuO}_{4}$ and $\mathrm{Ca}_{2} \mathrm{RuO}_{4}$ are reportedly tied to bias-controlled octahedral tilts ${ }^{55,56}$, and nanoIR imaging under simultaneous and correlative control of applied bias and uniaxial strain can evaluate this connection in real-space. In addition, owing to stronger spin-orbit coupling in $5 \mathrm{~d}$ transition metal oxides compared with their $4 \mathrm{~d}$ cousins, the resulting smaller Mott gaps in systems like $\mathrm{Sr}_{3} \mathrm{Ir}_{2} \mathrm{O}_{7}$ show exotic insulating and metallic phases strongly tunable through pressure ${ }^{57}$ with, we infer, remarkable real-space implications to resolve under applied uniaxial strain. However, we note that our strain-modulated technique is presently reliable only for samples of flat aspect ratio with uniform crystallinity and identifiable crystal orientation, somewhat constraining the eligibility of material systems for study. On the other hand, the high spatial resolution of infrared nano-imaging could foreseeably enable simultaneously straining and imaging uniform crystals as small as a few microns. In fact, suitable modifications to our strain device together with sample micro-manipulation could afford strain-modulated imaging of selected few-microns slices of single crystals, as isolated e.g. by focused ion beam milling.

Returning attention to our bilayer ruthenate, unexplored dimensions of the insulator-metal transition should be pursued by strain-modulated nano-imaging. Although we carried out these strain-modulated SNSImaging investigations on un-twinned CTRO crystals, the IMT in this system is also uncommon for its coupling to four-fold symmetry-breaking orthorhombic distortion. Owing to the bilayer unit cell, this ferroelastic distortion entails a polar moment oriented along the orthorhombic $b$-axis, rendering a hybrid-improper ferroelectric Mott insulator or high temperature "polar metal." 41,42,58 Its real-space implications for the IMT have hitherto gone completely unaddressed. We envision strainmodulated nano-imaging of insulator-metal domains near ferroelastic boundaries of twinned crystals, and whose manipulation might enable on-demand local re-orientation of striped domains, thus affording multi-functional control over this Mott nanotexture.

\section{METHODS}

\section{Multi-messenger low-temperature nano-imaging}

Scanning near-field optical microscopy (SNOM) is an atomic force microscope (AFM)-based technique enables imaging of surface optical properties at variable temperatures ${ }^{28,46,59}$ below the diffraction limit ${ }^{60}$, with a resolution strictly limited only by the geometric sharpness of the metallic AFM probe. We present $25 \mathrm{~nm}$-resolved imaging of the locally back-scattered near-field signal amplitude (abbreviated to nano-IR signal, or $\sigma$ ) collected at low temperatures (down to $T=50 \mathrm{~K}$ ) using a customdesigned cryogenic near-field optical microscope (cryo-SNOM) ${ }^{47}$. In these measurements, focused infrared light is incident upon and scattered from the metallic tip of an atomic force microscope (AFM) probe (SCM-PtSi, Bruker Nano) oscillating at a frequency $\sim 70 \mathrm{kHz}$ near the sample surface while the microscope is operated in amplitude modulation AFM feedback. The back-scattered radiation from the probe encodes information about the optical permittivity of the sample at the frequency of the laser source. Back-scattered radiation is registered by a liquid nitrogen-cooled mercury cadmium telluride photodetector and resolved from the background through Michelson interferometry in a pseudo-heterodyne detection scheme ${ }^{61}$. To a first approximation, the amplitude of back-scattered radiation modulated at high harmonics $(n \geq 2)$ of the cantilever oscillation frequency provides a proportional measure of the local near-field optical response of the sample and, by implication, its optical conductivity resolved at the 20-nm scale. However, previous works implicate further multiplicative influence from far-field reflectivity of the sample surrounding the probe, with possibility to distort the scale of even demodulated
nano-IR signals when scanning length scales of the sample comparable to the wavelength of illuminating radiation $(\sim 10 \text { microns })^{62}$. It is also recognized that nano-IR signals at higher harmonics $n$ provide increasingly strong contrast to material properties ${ }^{63}$. Thus, to remove the influence of far-field reflectivity, we take the nano-IR signal $\sigma$ as the ratio of demodulated back-scattered signals demodulated at harmonics $n=3$ and $n=2$, thus removing any commonly multiplicative "far-field factor."

Leveraging electrical conductivity of the nano-IR probe, for select images, Kelvin probe force detection is conducted simultaneously to nanoIR imaging using the frequency-modulation Kelvin probe force microscopy (FM-KPFM) method ${ }^{45}$. This method is acknowledged to afford artifact-free detection of the contact-potential difference, $\Delta \Phi=\Phi_{\text {CTRO }}-\Phi_{\text {tip }}$ in our case, resolved at length scales comparable to the tapping amplitude of the cantilevered probe. Approximating the work function of our KPFM probe by that for silicon $\left(\Phi_{\text {tip }} \approx 4.7 \mathrm{~V}\right)$, from data in Fig. If we can estimate the work function of metallic and insulating CTRO as $\Phi_{\text {CTRO }} \approx 4.89 \mathrm{~V}$ and $4.93 \mathrm{~V}$, respectively. These values are comparable to those encountered across the insulator-metal transition of other correlated oxides ${ }^{49}$.

\section{Variational treatment of the insulator-metal domain texture}

In the main text we treat the problem of phase evolution in a straincoupled insulator-metal transition using a quasi-one-dimensional variational model that provides an analytic understanding of thermal and elastic domain susceptibilities in CTRO. In this treatment, we consider a surficial layer of coexisting insulator and metal domains of depth $d$ with translational invariance along the crystal [100] axis, and denote by $x$ the position coordinate parallel to [010]. For simplicity we substitute the Landau free energy density articulated in Eq. (1) by the following $F_{\text {var }}$ per unit volume in the thickness $d$ :

$F_{\text {var }}(\psi)=\frac{1}{2} K|\nabla \psi|^{2}+F_{0}\left(g(T) \psi-\frac{1}{2} \psi^{2}+\frac{1}{2} \psi^{4}\right)-\delta \varepsilon K\left(\varepsilon_{\mathrm{ext}}+\varepsilon_{\text {int }}\right) \psi$

with $g(T) \propto\left(T-T_{C}\right) / \Delta T$ a surrogate measure of temperature, and other definitions given in the main text. The first two terms describe the electronic energy density $F_{\text {elec }}(\psi)$ and the last is the elastic energy density $F_{\text {elas }}(\psi)$. Given the linear coupling of $T$ and $\psi$ in this treatment, the elastic domain susceptibility $X_{\text {elas }}$ is manifestly proportional to the thermal one $X_{\text {therm }}$. Meanwhile, we determined $X_{\text {therm }}$ as presented in Fig. $4 \mathrm{c}$ from the temperature-dependent metallic phase fraction $f(T)$ estimated by a variational form for $\psi(x)$ through which we minimize Eq. (3):

$$
\begin{gathered}
\psi(x)=\psi_{M}+\frac{1}{2} \Delta \psi_{I, M} \sum_{n} \phi(x-n \lambda) \text { with } \\
\phi(x)=\tanh \left((x-f \lambda) / I_{d w}\right)-\tanh \left(x / I_{d w}\right) .
\end{gathered}
$$

Guided by our nano-imaging results, this form describes a onedimensional lattice of piecewise insulator and metal $\left(\psi=\psi_{I, M}\right)$ domains separated by domain walls of size $I_{d w}$ and with overall periodicity $\lambda$; the metallic phase fraction $f$ is manifestly represented. Including the energy of pairwise domain walls, the electronic energy density $F_{e l, \lambda}$ per unit period of this lattice is approximately:

$F_{\text {elec }, \lambda} \approx \frac{\kappa}{2 l_{d w}} \Delta \psi_{l, M}^{2}+\lambda\left[(1-f) F_{\text {elec }}\left(\psi_{l}\right)+f F_{\text {elec }}\left(\psi_{M}\right)+I_{d w} / \lambda \Delta F\right]$

where $\Delta F$ denotes height of the electronic energy barrier between insulator and metal phases pursuant to Eq. (3), and $I_{d w} \approx \frac{1}{2} \Delta \psi_{I, M} \sqrt{\kappa / \Delta F}$ can be asserted from the Landau theory of soliton domain walls ${ }^{64}$.

Lastly, setting $\varepsilon_{\text {ext }}=0$ for the equilibrium configuration, the elastic energy density per unit volume can be expressed:

$F_{\text {elas }}(\psi)=-d \frac{\delta \sigma^{2}}{2} \int_{-\infty}^{+\infty} d x \partial_{x} \psi(x) G_{x x}\left(x-x^{\prime}\right) \partial_{x^{\prime}} \psi\left(x^{\prime}\right)$

where $\delta \sigma \equiv K \delta \varepsilon$ is the $b$-axis dilatory component of spontaneous stress associated with the insulator-metal transition, and $G_{x x}$ is an elastic Green's function which for $\lambda>d$ we approximate ${ }^{17,50}$ by:

$G_{x x}(\vec{r}) \approx \frac{1+v}{\pi E}\left(\frac{1-v}{r}+v \frac{x^{2}}{r^{3}}\right)$ with $r=\sqrt{x^{2}+y^{2}}$.

Here $v \approx 0.3$ and $E$ are the Poisson's ratio and Young's modulus for CTRO, respectively, which we approximate using values reported for the analogous ruthenate $\mathrm{Sr}_{2} \mathrm{RuO}_{4}{ }^{65}$. Eq. (6) was evaluated by semi-analytic methods for our variational form $\phi(x)$. Taken together, Eq. (5-7) are sufficient to numerically determine the temperature-dependent metallic phase fraction $f(T, \lambda / d)$ that minimizes Eq. (1), from which we deduce $X_{\text {therm }}$ predicted in Fig. 4c. Further details and expressions for $f$ are 
provided in the Supplementary Discussion, which also discusses our provisions for the marginal cases at extremal temperatures where insulator or metal domains first nucleate with $(1-f) \lambda$ or $f \lambda \sim I_{d w}$.

\section{DATA AVAILABILITY}

All data presented in this work are available from the corresponding authors upon request.

Received: 1 August 2020; Accepted: 23 March 2021; Published online: 13 May 2021

\section{REFERENCES}

1. Dagotto, E. Complexity in strongly correlated electronic systems. Science 309, 257-262 (2005)

2. Milward, G. C., Calderón, M. J. \& Littlewood, P. B. Electronically soft phases in manganites. Nature 433, 607-610 (2005).

3. Dagotto, E. Nanoscale Phase Separation and Colossal Magnetoresistance vol. 136 Springer, Berlin (2003).

4. $\mathrm{Li}$, T. et al. Femtosecond switching of magnetism via strongly correlated spincharge quantum excitations. Nature 496, 69-73 (2013).

5. Imada, M., Fujimori, A. \& Tokura, Y. Metal-insulator transitions. Rev. Mod. Phys. 70 1039-1263 (1998).

6. Locquet, J. P. et al. Doubling the critical temperature of $\mathrm{La}_{1.9} \mathrm{Sr}_{0.1} \mathrm{CuO}_{4}$ using epitaxial strain. Nature 394, 453-456 (1998).

7. Burganov, B. et al. Strain control of Fermiology and many-body interactions in two-dimensional ruthenates. Phys. Rev. Lett. 116, 197003 (2016).

8. Lu, X. Z. \& Rondinelli, J. M. Epitaxial-strain-induced polar-to-nonpolar transitions in layered oxides. Nat. Mater. 15, 951-955 (2016).

9. Park, J. H. et al. Measurement of a solid-state triple point at the metal-insulator transition in $\mathrm{VO}_{2}$. Nature 500, 431-434 (2013).

10. $\mathrm{Wu}$, J. et al. Strain-induced self organization of metal-insulator domains in singlecrystalline $\mathrm{VO}_{2}$ nanobeams. Nano Lett. 6, 2313-2317 (2006).

11. Aetukuri, N. B. et al. Control of the metal-insulator transition in vanadium dioxide by modifying orbital occupancy. Nat. Phys. 9, 661-666 (2013).

12. Hepting, M. et al. Tunable charge and spin order in $\mathrm{PrNiO}_{3}$ thin films and superlattices. Phys. Rev. Lett. 113, 227206 (2014).

13. Dietl, $\mathrm{C}$. et al. Tailoring the electronic properties of $\mathrm{Ca}_{2} \mathrm{RuO}_{4}$ via epitaxial strain. Appl. Phys. Lett. 112, 031902 (2018).

14. Han, Q. \& Millis, A. Lattice energetics and correlation-driven metal-insulator transitions: the case of $\mathrm{Ca}_{2} \mathrm{RuO}_{4}$. Phys. Rev. Lett. 121, 067601 (2018).

15. Huang, $Z$. et al. Tuning the ground state of $\mathrm{La}_{0.67} \mathrm{Ca}_{0.33} \mathrm{MnO}_{3}$ films via coherent growth on orthorhombic $\mathrm{NdGaO}_{3}$ substrates with different orientations. Phys. Rev. B - Condens. Matter Mater. Phys. 86, 014410 (2012).

16. Zhang, J. et al. Cooperative photoinduced metastable phase control in strained manganite films. Nat. Mater. 15, 956-960 (2016).

17. McLeod, A. S. et al. Multi-messenger nanoprobes of hidden magnetism in a strained manganite. Nat. Mater. 19, 397-404 (2020).

18. Chu, J. H., Kuo, H. H., Analytis, J. G. \& Fisher, I. R. Divergent nematic susceptibility in an iron arsenide superconductor. Science 337, 710-712 (2012).

19. Kissikov, T. et al. Uniaxial strain control of spin-polarization in multicomponent nematic order of $\mathrm{BaFe}_{2} \mathrm{As}_{2}$. Nat. Commun. 9, 1058 (2018).

20. Bachmann, M. D. et al. Spatial control of heavy-fermion superconductivity in Celrln ${ }_{5}$. Science 366, 221-226 (2019).

21. Andrade, E. F. et al. Visualizing the nonlinear coupling between strain and electronic nematicity in the iron pnictides by elasto-scanning tunneling spectroscopy. arXiv 1812.05287 (2018).

22. Limelette, P. et al. Universality and critical behavior at the Mott transition. Science 302, 89-92 (2003).

23. Taniguchi, $\mathrm{H}$. et al. Anisotropic uniaxial pressure response of the Mott insulator $\mathrm{Ca}_{2} \mathrm{RuO}_{4}$. Phys. Rev. B - Condens. Matter Mater. Phys. 88, 205111 (2013).

24. Riccò, S. et al. In situ strain tuning of the metal-insulator-transition of $\mathrm{Ca}_{2} \mathrm{RuO}_{4}$ in angle-resolved photoemission experiments. Nat. Commun. 9, 4535 (2018).

25. Liu, M. K. et al. Anisotropic electronic state via spontaneous phase separation in strained vanadium dioxide films. Phys. Rev. Lett. 111, 096602 (2013).

26. Liu, M. et al. Symmetry breaking and geometric confinement in $\mathrm{VO}_{2}$ : results from a three-dimensional infrared nano-imaging. Appl. Phys. Lett. 104, 121905 (2014).

27. Pustogow, A., McLeod, A. S., Saito, Y., Basov, D. N. \& Dressel, M. Internal strain tunes electronic correlations on the nanoscale. Sci. Adv. 4, eaau9123 (2018).

28. McLeod, A. S. et al. Nanotextured phase coexistence in the correlated insulator $\mathrm{V}_{2} \mathrm{O}_{3}$. Nat. Phys. 13, 80-86 (2017).
29. Baumberger, F. et al. Nested Fermi surface and electronic instability in $\mathrm{Ca}_{3} \mathrm{Ru}_{2} \mathrm{O}_{7}$ Phys. Rev. Lett. 96, 107601 (2006).

30. Lee, J. S. et al. Pseudogap dependence of the optical conductivity spectra of $\mathrm{Ca}_{3} \mathrm{Ru}_{2} \mathrm{O}_{7}$ - a possible contribution of the orbital flip excitation. Phys. Rev. Lett. 98 097403 (2007).

31. $\mathrm{Ke}, \mathrm{X}$. et al. Emergent electronic and magnetic state in $\mathrm{Ca}_{3} \mathrm{Ru}_{2} \mathrm{O}_{7}$ induced by $\mathrm{Ti}$ doping. Phys. Rev. B - Condens. Matter Mater. Phys. 84, 201102(R) (2011).

32. Peng, J. et al. From quasi-two-dimensional metal with ferromagnetic bilayers to Mott insulator with G-type antiferromagnetic order in $\mathrm{Ca}_{3}\left(\mathrm{Ru}_{1-x} \mathrm{Ti}_{\mathrm{x}}\right)_{2} \mathrm{O}_{7}$. Phys. Rev. B - Condens. Matter Mater. Phys. 87, 085125 (2013).

33. Barker, B. I. et al. STM studies of individual Ti impurity atoms in $\mathrm{Sr}_{2} \mathrm{RuO}_{4}$. Phys. $B$ Condens. Matter 329-333, 1334-1335 (2003).

34. Lee, J. et al. Heavy d-electron quasiparticle interference and real-space electronic structure of $\mathrm{Sr}_{3} \mathrm{Ru}_{2} \mathrm{O}_{7}$. Nat. Phys. 5, 800-804 (2009)

35. Peng, J. et al. Mott transition controlled by lattice-orbital coupling in $3 \mathrm{~d}$-metaldoped double-layer ruthenates. Phys. Rev. B 96, 205105 (2017).

36. Salje, E. K. H. Ferroelastic materials. Annu. Rev. Mater. Res. 42, 265-283 (2012).

37. Yoshida, Y. et al. Crystal and magnetic structure of $\mathrm{Ca}_{3} \mathrm{Ru}_{2} \mathrm{O}_{7}$. Phys. Rev. $B-$ Condens. Matter Mater. Phys. 72, 054412 (2005).

38. Zhu, M. et al. Colossal magnetoresistance in a Mott insulator via magnetic fielddriven insulator-metal transition. Phys. Rev. Lett. 116, 216401 (2016).

39. Zou, T. et al. Pressure-induced electronic and magnetic phase transitions in a Mott insulator: Ti-doped $\mathrm{Ca}_{3} \mathrm{Ru}_{2} \mathrm{O}_{7}$ bilayer ruthenate. Phys. Rev. B 94, 041115(R) (2016).

40. Stone, G. et al. Atomic and electronic structure of domains walls in a polar metal. Phys. Rev. B 99, 014105 (2019).

41. Lei, S. et al. Observation of quasi-two-dimensional polar domains and ferroelastic switching in a metal, $\mathrm{Ca}_{3} \mathrm{Ru}_{2} \mathrm{O}_{7}$. Nano Lett. 18, 3088-3095 (2018).

42. Oh, Y. S., Luo, X., Huang, F. T., Wang, Y. \& Cheong, S. W. Experimental demonstration of hybrid improper ferroelectricity and the presence of abundant charged walls in $(\mathrm{Ca}, \mathrm{Sr})_{3} \mathrm{Ru}_{2} \mathrm{O}_{7}$ crystals. Nat. Mater. 14, 407-413 (2015).

43. Rondinelli, J. M. \& Fennie, C. J. Octahedral rotation-induced ferroelectricity in cation ordered perovskites. Adv. Mater. 24, 1961-1968 (2012).

44. Gangshettiwar, A. et al. Emergence of a competing stripe phase near the Mott transition in Ti-doped bilayer calcium ruthenates. Phys. Rev. B 101, 201106(R) (2020)

45. Melitz, W., Shen, J., Kummel, A. C. \& Lee, S. Kelvin probe force microscopy and its application. Surf. Sci. Rep. 66, 1-27 (2011).

46. Qazilbash, M. M. et al. Mott transition in $\mathrm{VO}_{2}$ revealed by infrared spectroscopy and nano-imaging. Science 318, 1750-1753 (2007).

47. Post, K. W. et al. Coexisting first- and second-order electronic phase transitions in a correlated oxide. Nat. Phys. 14, 1056-1061 (2018).

48. Liu, M. et al. Phase transition in bulk single crystals and thin films of $\mathrm{VO}_{2}$ by nanoscale infrared spectroscopy and imaging. Phys. Rev. B - Condens. Matter Mater. Phys. 91, 245155 (2015).

49. Sohn, A., Kanki, T., Sakai, K., Tanaka, H. \& Kim, D. W. Fractal nature of metallic and insulating domain configurations in a $\mathrm{VO}_{2}$ thin film revealed by Kelvin probe force microscopy. Sci. Rep. 5, 1-7 (2015).

50. Zhang, J. et al. Nano-resolved current-induced insulator-metal transition in the mott insulator $\mathrm{Ca}_{2} \mathrm{RuO}_{4}$. Phys. Rev. X 9, 11032 (2019).

51. Khachaturyan, A. G. Habit plane and orientation relations in precipitates: comparison with experimental data. in Theory of Structural Transformations in Solids 299-305 (2013).

52. Mester, L., Govyadinov, A. A., Chen, S., Goikoetxea, M. \& Hillenbrand, R. Subsurface chemical nanoidentification by nano-FTIR spectroscopy. Nat. Commun. 11, 3359 (2020)

53. Miao, L. et al. Itinerant ferromagnetism and geometrically suppressed metalinsulator transition in epitaxial thin films of $\mathrm{Ca}_{2} \mathrm{RuO}_{4}$. Appl. Phys. Lett. 100, 052401 (2012).

54. Sae Hong, S. et al. Two-dimensional limit of crystalline order in perovskite membrane films. Sci. Adv. 3, 1-6 (2017).

55. Cao, G., Bolivar, J., McCall, S., Crow, J. \& Guertin, R. Weak ferromagnetism, metalto-nonmetal transition, and negative differential resistivity in single-crystal $\mathrm{Sr}_{2} \mathrm{IrO}_{4}$. Phys. Rev. B - Condens. Matter Mater. Phys. 57, R11039(R) (1998).

56. Nakamura, F. et al. Electric-field-induced metal maintained by current of the Mott insulator $\mathrm{Ca}_{2} \mathrm{RuO}_{4}$. Sci. Rep. 3, 2536 (2013).

57. Ding, Y. et al. Pressure-induced confined metal from the Mott insulator $\mathrm{Sr}_{3} \mathrm{Ir}_{2} \mathrm{O}_{7}$ Phys. Rev. Lett. 116, 216402 (2016).

58. Benedek, N. A. \& Fennie, C. J. Hybrid improper ferroelectricity: a mechanism for controllable polarization-magnetization coupling. Phys. Rev. Lett. 106, 107204 (2011).

59. Yang, H. U., Hebestreit, E., Josberger, E. E. \& Raschke, M. B. A cryogenic scatteringtype scanning near-field optical microscope. Rev. Sci. Instrum. 84, 23701-101124 (2013). 
60. Atkin, J. M., Berweger, S., Jones, A. C. \& Raschke, M. B. Nano-optical imaging and spectroscopy of order, phases, and domains in complex solids. Adv. Phys. 61, 745-842 (2012).

61. Ocelic, N., Huber, A. \& Hillenbrand, R. Pseudoheterodyne detection for background-free near-field spectroscopy. Appl. Phys. Lett. 89, 101124 (2006).

62. Zhang, L. M. et al. Near-field spectroscopy of silicon dioxide thin films. Phys. Rev. B - Condens. Matter Mater. Phys. 85, 075419 (2012).

63. Knoll, B. \& Keilmann, F. Enhanced dielectric contrast in scattering-type scanning near-field optical microscopy. Opt. Commun. 182, 321-328 (2000).

64. Sanati, M. \& Saxena, A. Landau theory of domain walls for one-dimensional asymmetric potentials. Am. J. Phys. 71, 1005-1012 (2003).

65. Paglione, J. et al. Elastic tensor of $\mathrm{Sr}_{2} \mathrm{RuO}_{4}$. Phys. Rev. B - Condens. Matter Mater. Phys. 65, 2205061-2205064 (2002)

\section{ACKNOWLEDGEMENTS}

Work on correlated oxides at Columbia was supported entirely by the Center on Precision-Assembled Quantum Materials, funded through the US National Science Foundation (NSF) Materials Research Science and Engineering Centers (award no. DMR-2011738). D.N.B. is Moore Investigator in Quantum Materials EPIQS \#9455. D.N.B. is the Vannevar Bush Faculty Fellow ONR-VB: N00014-19-1-2630. L.-Q.C., V.G. and Y.Y. acknowledge support from the US Department of Energy, Office of Science, Office of Basic Energy Sciences, under Award Number DE-SC-0012375 and the partial support from the US Department of Energy, Office of Science, Basic Energy Sciences, under Award Number DE-SC0020145 as part of the Computational Materials Sciences Program. Z.Q.M. and V.G. acknowledge financial support for sample preparation provided by the National Science Foundation through the Penn State 2D Crystal Consortium-Materials Innovation Platform (2DCC-MIP) under NSF cooperative agreement DMR-1539916. Work on preparing strain devices was supported by the Air Force Office of Scientific Research via grant FA9550-16-1-0601.

\section{AUTHOR CONTRIBUTIONS}

A.S. McLeod and A. Wieteska devised and carried out the experiments and share the first authorship of this work. A.S. McLeod, F. Xue, and L.Q. Chen carried out the numerical simulations. B. Foutty, Y. Yuan, and V. Gopalan provided material support for the experiments. G. Chiriaco and A.J. Millis devised the variational elastic Landau theory. Y. Wang and Z. Mao provided the single crystals under study. A. Pasupathy and D.N. Basov oversaw these studies. A.S. McLeod wrote the manuscript together with A. Wieteska and with strong input from all co-authors.

\section{COMPETING INTERESTS}

The authors declare no competing interests.

\section{ADDITIONAL INFORMATION}

Supplementary information The online version contains supplementary material available at https://doi.org/10.1038/s41535-021-00339-0.

Correspondence and requests for materials should be addressed to A.S.M. or A.N.P.

Reprints and permission information is available at http://www.nature.com/ reprints

Publisher's note Springer Nature remains neutral with regard to jurisdictional claims in published maps and institutional affiliations.

(i) Open Access This article is licensed under a Creative Commons Attribution 4.0 International License, which permits use, sharing, adaptation, distribution and reproduction in any medium or format, as long as you give appropriate credit to the original author(s) and the source, provide a link to the Creative Commons license, and indicate if changes were made. The images or other third party material in this article are included in the article's Creative Commons license, unless indicated otherwise in a credit line to the material. If material is not included in the article's Creative Commons license and your intended use is not permitted by statutory regulation or exceeds the permitted use, you will need to obtain permission directly from the copyright holder. To view a copy of this license, visit http://creativecommons. org/licenses/by/4.0/.

(c) The Author(s) 2021 